\title{
A study on the rearrangement corrections to the folding model applied to nucleon inelastic scattering
}

\author{
M. Dupuis ${ }^{1, a}$ and E. Bauge ${ }^{1}$ \\ ${ }^{1}$ CEA, DAM, DIF, 91297 Arpajon Cedex
}

\begin{abstract}
A study of direct neutron induced reactions off spherical nuclei is given in terms of a folding model. Matter densities stemming from the Random Phase Approximation (RPA), implemented with the Gogny D1S force are used to provide the relevant coupling potentials. The impact of rearrangement corrections on cross sections is studied for direct inelastic nucleon scattering off ${ }^{16} \mathrm{O}$ and ${ }^{208} \mathrm{~Pb}$. The rearrangement correction to calculated cross sections is shown to strongly depend on the incident energy and on the excitation multipolarity.
\end{abstract}

\section{Introduction}

Energy application and astrophysical models require precise nuclear data for a large range of nuclear masses. The development of microscopic models is necessary to fulfill this need, since such models are able to predict cross sections when nuclear measurement are hard or impossible to achieve, and consequently, when phenomenological models are not well constrained.

We aim at reducing phenomenology in the description of nucleon induced reaction, focusing on the direct inelastic scattering process using a microscopic description. Works along this line can be performed in the framework of the Jeukenne-Lejeune-Mahaux (JLM) folding model [1] to describe nucleon elastic and inelastic scattering off both spherical and deformed targets using densities stemming from mean-field and beyond mean field calculations [2-4]. Besides, Cheon et al. [5, 6] pointed out that a simple identification of the g-matrix to the effective interaction is invalid when applied to nucleon inelastic scattering process calculations, and that an additional density-dependent term, the rearrangement potential, appears. This correction accounts for the fluctuation of the density during the transition which thus modifies the interaction. For this reason, this approach is also often called consistent or dynamic treatment of inelastic scattering. An expression of the rearrangement correction to the folding model transition potential was first obtained in [5], using the vibrational model approach that identifies the transition potential shape to the derivative of the optical potential. The rearrangement expression was latter justified from a more fundamental point of view in [6] in the framework of the Watson multiple scattering theory, in the zero momentum transfer limit, for a simple particle-hole excitation. These early works showed that the rearrangement correction has a non-negligible impact on calculated nucleon differential inelastic cross sections. This correction has then been used in some [7-9] yet not all applications of folding models based on various nuclear matter calculations, such as

ae-mail: marc.dupuis@cea.fr 
the density dependent M3Y interactions [10], the Melbourne g-matrix [11, 12] or the JLM approach [4]. However, when the rearrangement corrections were used, their impact on direct inelastic cross section has been rarely studied. We only account for the work of El-Azab Farid and Satchler [7], performed with a density dependent M3Y interaction, that reports a reduction of $28 \%$ of the $65 \mathrm{MeV}$ proton inelastic scattering off ${ }^{208} \mathrm{~Pb}$ to the $3_{1}^{-}$states due to the rearrangement. They showed some important change of $\alpha$ scattering cross sections related to the consistency requirement suggested by Cheon et al. Recently, a study of direct inelastic neutron scattering off spherical and axially deformed targets was performed within the JLM folding model [13] with nuclear matter densities obtained from the Quasi-Particle Random Phase Approximation (QRPA) with the Gogny D1S force [14, 15]. In this work, rearrangement corrections to the inelastic cross section were explicitly shown only for the $2_{1}^{+}$ excitation in ${ }^{208} \mathrm{~Pb}$ for medium energy neutron scattering $(E \simeq 7-20 \mathrm{MeV})$. For this transition at these incident energies, rearrangement was found to reduce the magnitudes of inelastic scattering differential cross sections by $50 \%$. In the present paper, we focus on the characterization of the rearrangement correction to nucleon direct inelastic scattering off spherical nuclei in the context of the JLM folding model. We extend the impact analysis of rearrangement on direct inelastic cross sections of ref. [13] to a wider incident energy range, for both neutron and proton scattering, and for various excitation multipolarities.

The first section provides the main features of the model. A characterization of the rearrangement corrections for nucleon scattering off spherical nuclei is given in the second section. Conclusions are drawn in the last section.

\section{JLM folding model for shape elastic and direct inelastic scattering}

All details about the JLM folding model for elastic and direct inelastic nucleon scattering can be found in $[3,13]$ (and references therein), so we only remind here its main features. It is based on a Brueckner Hartree-Fock derivation of the optical potential for nucleon scattering in nuclear matter (NM). For a nucleon incident energy $E$ and neutron and proton density $\rho_{n}, \rho_{p}$, Jeukenne et al. expressed their NM optical potential as:

$$
\begin{aligned}
V_{\tau}^{\mathrm{JLMB}}\left(\rho_{n}, \rho_{p}, E\right)=\lambda_{V}(E)[ & \left.V_{0}(\rho, E)+\tau \lambda_{V_{1}}(E) \alpha\left(\rho_{n}, \rho_{p}\right) V_{1}(\rho, E)\right] \\
& +i \lambda_{W}(E)\left[W_{0}(\rho, E)+\tau \lambda_{W_{1}}(E) \alpha\left(\rho_{n}, \rho_{p}\right) W_{1}(\rho, E)\right] .
\end{aligned}
$$

where $\alpha\left(\rho_{n}, \rho_{p}\right)=\frac{\rho_{n}-\rho_{p}}{\rho_{n}+\rho_{p}}, \tau=+1(-1)$ for neutron (proton) scattering. The energy dependent parameters $\lambda_{V}, \lambda_{V_{1}}, \lambda_{W}, \lambda_{W_{1}}$ are provided in $[3,16]$.

An optical potential for nucleon scattering off a finite nucleus can be determined from this NM optical potential using the so-called improved local density approximation (LDA) [1].

Following the work of Cheon et al. [5,6], the transition potential for direct nucleon inelastic scattering can be identified to the derivative of the optical potential with respect to the isoscalar and isovector matter densities. In the context of the JLM folding model, the transition potentials that include rearrangement were specified in [13]. We only remind here their main expressions, where dependencies other than densities are not explicitly written. The transition potential corresponding to a nucleon scattering off a target in its ground state and leading to a discrete excitation $|N\rangle$ reads

$$
U=U^{(0)}+U^{(R)}
$$


where $U^{(0)}$ is the usual folding transition potential and $U^{(R)}$ corresponds to the rearrangement correction. These potentials read

$$
\begin{aligned}
U^{(0)} & =\rho^{(0, N)} F_{0}(\rho)+\tau \rho_{1}^{(0, N)} F_{1}(\rho), \\
U^{(R)} & =\rho^{(0, N)}\left(\rho \frac{\partial F_{0}(\rho)}{\partial \rho}+\tau \rho_{1} \frac{\partial F_{1}(\rho)}{\partial \rho}\right),
\end{aligned}
$$

where $\rho^{(0, N)}=\rho_{n}^{(0, N)}+\rho_{p}^{(0, N)}$ and $\rho_{1}^{(0, N)}=\rho_{n}^{(0, N)}-\rho_{p}^{(0, N)}$. The $\rho_{n / p}^{(0, N)}$ are the radial transition densities $\rho_{n / p}^{(0, N)}(\mathbf{r})=\sum_{\sigma}\left\langle N\left|\Psi^{\dagger}(\mathbf{r}, \sigma, t) \Psi(\mathbf{r}, \sigma, t)\right| \tilde{0}\right\rangle$ with $t=\frac{1}{2}\left(-\frac{1}{2}\right)$ for neutron (proton). The factors $F_{0}$ and $F_{1}$ reads:

$$
F_{0}(\rho)=\lambda_{V} V_{0}(\rho)+i \lambda_{W} W_{0}(\rho) \quad, \quad F_{1}(\rho)=\frac{1}{\rho}\left[\lambda_{V} \lambda_{V_{1}} V_{1}(\rho)+i \lambda_{W} \lambda_{W_{1}} W_{1}(\rho)\right]
$$

\section{Rearrangement corrections in spherical nuclei}

In [5], Cheon et al. displayed the impact of the rearrangement term on the differential cross section for the reaction $135 \mathrm{MeV}{ }^{16} \mathrm{O}\left(\mathrm{p}, \mathrm{p}\right.$ ') to the $3_{1}^{-}$excitation located at $E_{x}=6.13 \mathrm{MeV}$. In this study, the von Geramb's g-matrix [5] and phenomenological transition densities were used. Here, we perform an equivalent analysis within the JLM folding model described in the previous section and RPA transition densities [17] calculated with the Gogny D1S interaction. Note that, for $3_{1}^{-}$excitation, the RPA/D1S model provides the reduced electric transition probability $B^{R P A}(E 3 \uparrow)=0.125\left(10^{4} e^{2} \mathrm{fm}^{6}\right)($ calculation performed in an harmonic oscillator basis with 11 major shells) as compared to the adopted value $B^{\exp .}(E 3 \uparrow)=0.1485\left(10^{4} e^{2} \mathrm{fm}^{6}\right)$ [18]. The theoretical transition density used in the folding has thus been multiplied by the factor $\sqrt{\frac{B^{\exp p}}{B^{R P A}}}=1.088$ to account for this discrepancy. Cross sections were calculated, within the distorted wave Born approximation, using either the transition potential $U=U^{(0)}+U^{(R)}$, eq. (2), that includes rearrangement corrections, or the transition potential $U^{(0)}$, eq. (3), without these corrections. The results displayed on Fig.1 (left panel) follow those reported by Cheon et al.: for this transition at this incident energy, the rearrangement correction reduces the differential cross section at forward angles by an amount of $15 \%$. We extend this study to determine the magnitude of the rearrangement correction for neutron/proton scattering in the incident energy range 10-200 MeV. We display on Fig. 1 (right panel) the ratio of the angular integrated inelastic cross section $\sigma$, calculated with the transition potential $U=U^{(0)}+U^{(R)}$, to the one calculated with the transition potential $U^{(0)}$, labeled $\sigma_{(0)}$. For proton scattering (full black curve), the rearrangement correction impacts the most on inelastic cross section at the lowest and highest incident energies. It reduces the inelastic cross section magnitude by $40 \%$ at $E=10 \mathrm{MeV}$ and $25 \%$ at $E=200 \mathrm{MeV}$. The smallest correction is found at $E \simeq 135 \mathrm{MeV}$. We note that the proton ratio is shifted to the right with respect to the neutron ratio by 5 to $10 \mathrm{MeV}$. This is only the consequence of the Coulomb barrier undergone by protons.

We now extend the previous study to neutron and proton scattering off ${ }^{208} \mathrm{~Pb}$ using ground state and transition densities predicted by the RPA/D1S nuclear structure model (calculation performed in an harmonic oscillator basis with 15 major shells). Figure 2 (left panel) illustrates the rearrangement renormalization effect on angular distributions for proton inelastic scattering to the $2_{1}^{+}$states that lies at $4.085 \mathrm{MeV}$. The RPA/D1S predicted value is $B^{R P A}(E 2 \uparrow)=0.293\left(10^{4} e^{2} \mathrm{fm}^{4}\right)$, in agreement with the adopted value $B^{\text {exp. }}(E 2 \uparrow)=0.30030\left(10^{4} e^{2} \mathrm{fm}^{4}\right)$ [26]. The ratios $\frac{\sigma}{\sigma_{(0)}}$ in the incident energy range 7-200 MeV for natural parity Yrast excitations with spin $J=2,4,6$ and $8 \hbar$ are displayed in Figure 2 

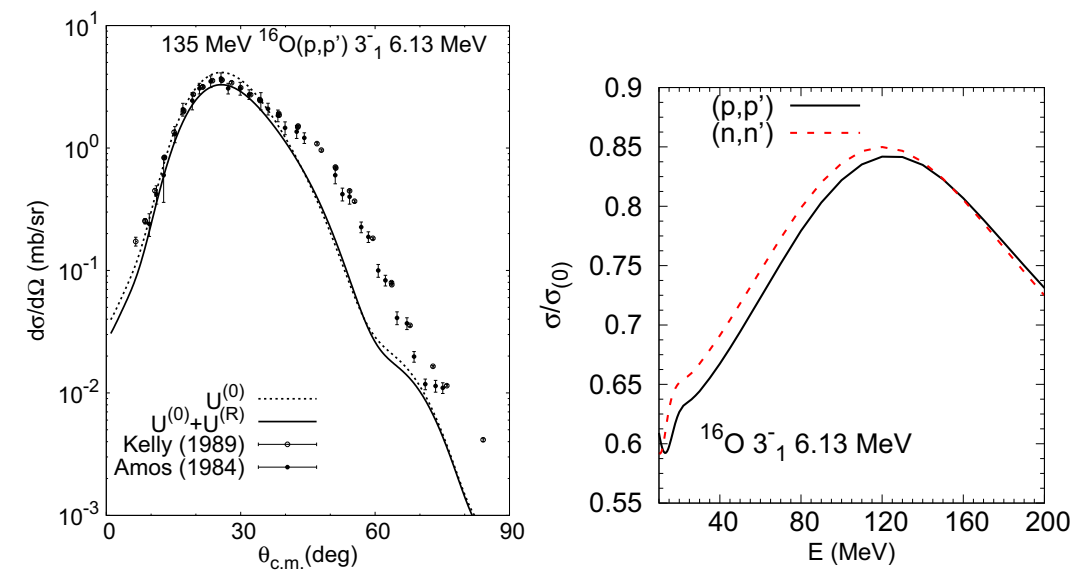

Figure 1. Left panel: differential inelastic cross section for $\mathrm{p}+{ }^{16} \mathrm{O}$ at the incident energy $135 \mathrm{MeV}$ for the $3_{1}^{-}$excitation at $6.13 \mathrm{MeV}$ (data from $[19,20]$ ). Full (dashed) black curve: cross section calculated from the transition potential $U=U^{(0)}+U^{(R)}$, $\left(U^{(0)}\right)$, defined in eqs. (2) to (4). Right panel: direct inelastic cross section ratios $\frac{\sigma}{\sigma_{(0)}}$ for the $3_{1}^{-}$excitation (see details in the text) for $\mathrm{p} / \mathrm{n}+{ }^{16} \mathrm{O}$ (full black / red dashed curve) in the incident energy range $10-200 \mathrm{MeV}$.

(right panel). At low energy, the ratio for proton scattering (full black curve in the upper panel) moves rapidly towards unity since the inelastic scattering process is essentially due to Coulomb excitation below the Coulomb barrier. The energy dependence of the cross section ratio is qualitatively the same as the one observed for ${ }^{16} \mathrm{O}$, but the renormalization effect is stronger, and its minimum is found at $110 \mathrm{MeV}$, as compared to $135 \mathrm{MeV}$ in the ${ }^{16} \mathrm{O}$ case. This minimum is shifted towards higher energies when the spin of the transition increases, and the magnitude of the rearrangement correction strongly decreases when the spin of the transition increases. As medium energy nucleons mainly probe the nucleus surface, the strong decrease of the inelastic cross section can be related to the reduction of the transition potential at the nuclear surface, caused by the rearrangement correction [13]. To interpret the evolution of the rearrangement impact on the inelastic cross section in the whole energy range and for the various transitions displayed in Figs.1 and 2, we would need to consider two features. First, both the real and imaginary potentials $U^{(0)}$ and $U^{(R)}$ strongly depend on the incident energy, as illustrated in Fig. 3, and on the multipolarity of the excitation. Second, different radial extents of the potentials are probed by the projectile according to the incident energy in such a way that various contributions can produce constructive or destructive interferences. In [7] an analysis of $65 \mathrm{MeV}$ proton inelastic scattering off ${ }^{208} \mathrm{~Pb}$ to the $3_{1}^{-}$states and to the giant monopole resonance revealed that strong interferences occur between the contribution to the inelastic cross section of the inner and outer parts of the transition potentials. This analysis will be extended to the energy range and transitions displayed in the present study. This will be reported in a forthcoming paper.

\section{Conclusion}

We report on microscopic calculations of direct inelastic scattering cross sections for nucleon induced reactions off ${ }^{16} \mathrm{O},{ }^{208} \mathrm{~Pb}$ in the framework of the JLM folding model. Rearrangement impact on direct inelastic cross sections is shown to strongly depend on the incident energy and the transition multipolarities. An analysis of the relevant transition potentials will be performed to interpret this behavior 

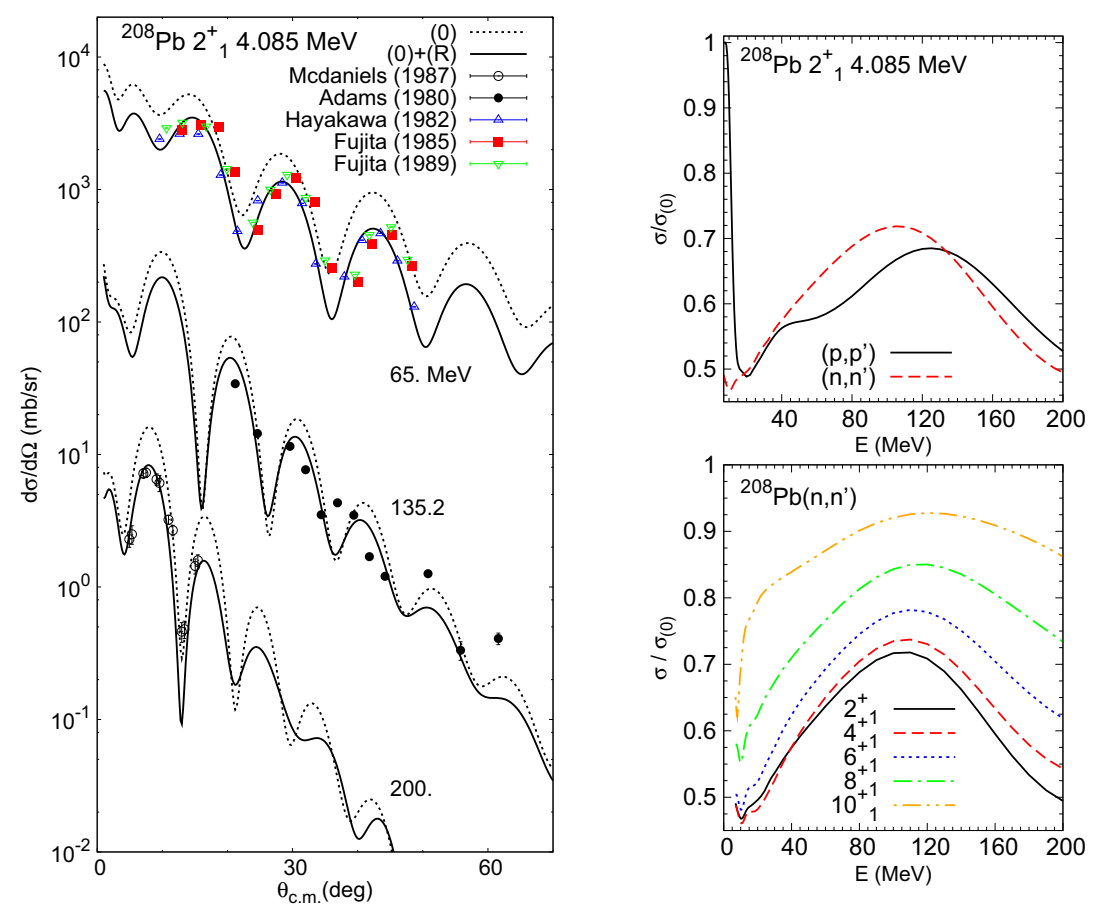

Figure 2. Left panel: differential inelastic cross sections for $\mathrm{p}+{ }^{208} \mathrm{~Pb}$ at incident energies 65 (data from [21-23], 135 (data from [24]) and $200 \mathrm{MeV}$ (data from [25]) for the $2_{1}^{+}$excitation lying at $4.065 \mathrm{MeV}$. Full (dashed) black curves: cross sections calculated from the transition potential $U=U^{(0)}+U^{(R)},\left(U^{(0)}\right)$, defined in eqs. (2) to (4). Top right panel: inelastic cross section ratios $\frac{\sigma}{\sigma_{(0)}}$ for the $2_{1}^{+}$excitation (see details in the text) for $\mathrm{p} / \mathrm{n}+{ }^{208} \mathrm{~Pb}$ (full black / red dashed curve) in the incident energy range 7-200 MeV. Bottom right panel: same ratios for neutron inelastic scattering to the $2_{1}^{+}, 4_{1}^{+}, 6_{1}^{+}, 8_{1}^{+}$and $10_{1}^{+}$states.
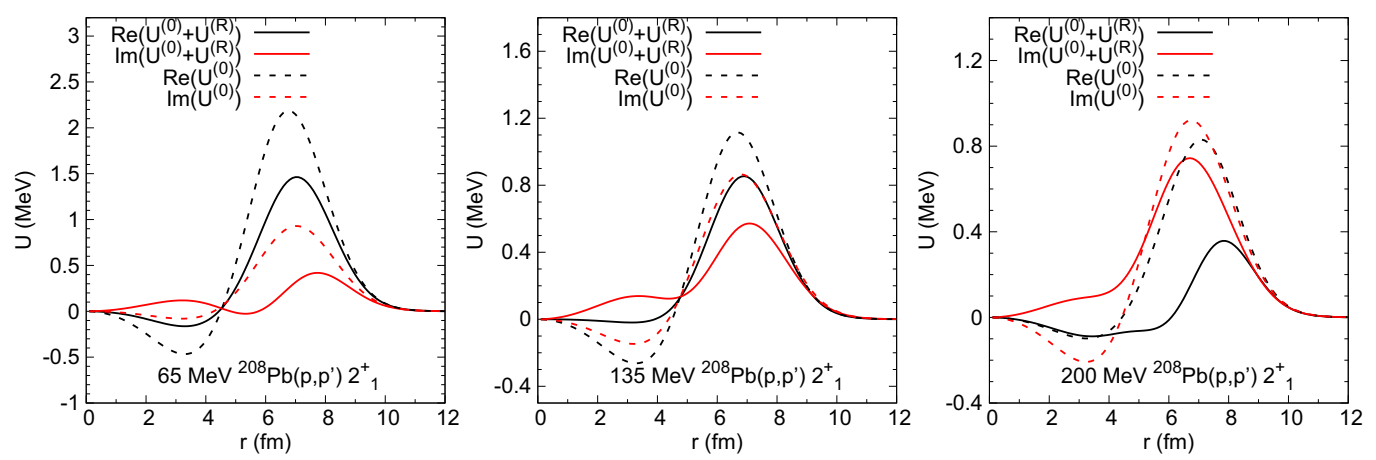

Figure 3. Transition potentials for proton inelastic scattering off ${ }^{208} \mathrm{~Pb}$ to the $2_{1}^{+}$state at incident energies 65 , 135.2 and $200 \mathrm{MeV}$. Real and imaginary parts of the transition potential $U=U^{(0)}+U^{(R)}$, and $U^{(0)}$, defined in eqs. (2) to (4), are plotted. 
for transitions in both spherical and axially deformed nuclei using QRPA $[14,15]$ nuclear structure input. It appears, at least for the reactions studied here and those reported in [13], that following Cheon et al.'s prescription for rearrangement [5] improves the agreement between calculated direct inelastic cross sections and measurements. Note that care should be taken to avoid any double counting of diagrams, such as the second order diagram in the hole line (low density) expansion which could be identified to rearrangement diagrams which are exemplified in [6], and that may be already included in the nuclear matter calculation. The JLM folding model detailed in [1] is based on an optical potential calculated in nuclear matter within the Brueckner Hartree-Fock approximation. Thus second order diagrams in the hole line expansion, such as those described in [27], are not included and the rearrangement corrections of $[5,6]$ seem to be appropriate in applications with the JLM folding model for vibrational, spin independent, collective states. However, more fundamental work is still needed to clearly establish the expressions of the rearrangement term in the most general case. This will be most certainly helpful in determining which correction needs to be introduced in folding models used in direct nucleon or ion inelastic scattering studies for excitations of various nature, and effective interactions based on various nuclear matter calculations.

\section{References}

[1] J.P. Jeukenne, A. Lejeune, C. Mahaux, Phys. Rev. C 16 (1977)

[2] E. Bauge, J. Delaroche, M. Girod, Nucl. Phys. A 654 (1999)

[3] E. Bauge, J.P. Delaroche, M. Girod, Phys. Rev. C 63 (2001)

[4] E. Khan, et al., Nucl. Phys. A 694, 103 (2001)

[5] T. Cheon, K. Takayanagi, K. Yazaki, Nucl. Phys. A 437, 301 (1985)

[6] T. Cheon, K. Takayanagi, K. Yazaki, Nucl. Phys. A 445, 227 (1985)

[7] M. El-Azab Farid, G. Satchler, Nucl. Phys. A 481, 542 (1988)

[8] D.T. Khoa, G. Satchler, Nucl. Phys. A 668 (2000)

[9] D.T. Khoa, E. Khan, G. Colò, N.V. Giai, Nucl. Phys. A 706 (2002)

[10] M. Dupuis, T. Kawano, J.P. Delaroche, E. Bauge, Phys. Rev. C 83 (2011)

[11] M. Dupuis, S. Karataglidis, E. Bauge, J.P. Delaroche, D. Gogny, Phys. Lett. B 665 (2008)

[12] K. Amos, et al., Adv. in Nucl. Phys. 25 (2000)

[13] M. Dupuis, et al., Eur. Phys. J. A 51 (2015)

[14] S. Péru, H. Goutte, Phys. Rev. C 77 (2008)

[15] S. Péru, G. Gosselin, M. Martini, M. Dupuis, S. Hilaire, J.C. Devaux, Phys. Rev. C 83 (2011)

[16] S. Goriely, J.P. Delaroche, Phys. Lett. B 653 (2007)

[17] J. Blaizot, D. Gogny, Nuc. Phys. A 284, 429 (1977)

[18] T. Kibédi, R. Spear, Atomic Data and Nuclear Data Tables 80, 35 (2002)

[19] K. Amos, et al., Nucl. Phys. A 413 (1984)

[20] J. J. Kelly, et al., Phys. Rev. C 39 (1989)

[21] S. I. Hayakawa, et al., Phys. Rev. Let. 49 (1982)

[22] Y. Fujita, et al., Phys. Rev. C 32 (1985)

[23] Y. Fujita, et al., Phys. Rev. C 40 (1989)

[24] G. S. Adams, et al., Phys. Lett. B 91 (1980)

[25] D. K. Mcdaniels, et al., Nuc. Phys. A 467 (1987)

[26] S. Raman, C. Nestor Jr., P. Tikkanen, Atomic Data and Nuclear Data Tables 78, 1 (2001)

[27] J.P. Jeukenne, A. Lejeune, C. Mahaux, Phys. Rep. 25 (1976) 